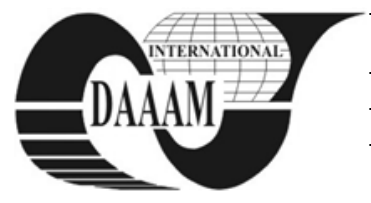

\title{
POSSIBILITIES OF DATA EXCHANGE BETWEEN MODELS OF PRODUCTION SYSTEMS
}

\author{
NOVAK, I[vo]
}

\begin{abstract}
This paper focuses on production systems modelling and especially on data exchange between manufacturers' system models within supply chain. Solutions oriented on easy operation of simulation software for people with minimum knowledge are presented. Moreover there are methods for model creation introduced. These methods are focused on computed load reduction. Two ways of model creating are introduced - simulation time and order size dependent.

Key words: computer simulation, plant simulation, MS Office Excel, data exchange model
\end{abstract}

\section{INTRODUCTION}

Product finalization is commonly provided through cooperation of several suppliers. The value chain requires effective management and ability to predict the amount of production, in order to establish optimal performance. One of the options for optimizations, are computer simulations. Software is often used for individual manufacturing systems optimization. However this article is focused on other options specifically on effective data exchange between models of production systems of individual subcontractors. This article also deals with methods oriented on simplification of operator skill needs and on reduction of simulation time needs for purposes of efficiency improvement. Software Plant Simulation from Siemens is used.

\section{EFFECTIVE DATA EXCHANGE BETWEEN MODELS}

Plant simulation software offers communication linkage with Excel. The linkage is used because of the extensive usage of Excel software and its well-known functionalities. Computer simulations require only basic Excel knowledge.

Some authors mention functionalities that provide statistical data transfer into Excel, after simulation procedures are terminated (e.g. Kumar, Nottestad, Macklin (2007), Lassalle, Wang, Owen, Mileham(2007)). The linkage is used for processing large amount of input and output data. Particularly Lassalle, Wang, Owen, Mileham(2007) describe the linkage involving different types of transferred information.

In our case, we assume several models of individual manufacturing systems and we want to ensure an exchange of data about the number of manufactured products. In other words, after each simulation, data have to be delivered to next model. Then the following model automatically uploads the data. After the simulation process is finished, the data is transfered further. In order to establish such a flow, the PLANT SIMULATION software functions have been adjusted. Standard program setup usually records number of manufactured products into Excel. However this recording is conducted after each manufactured product. Demand factor for simulation time is fairly high, see tab. 1 (chapter 2.3).
Therefore, the program needs to be adjusted, in order to record data only at time neccesary to provide valid simulation result (not each time a product is manufactured).

The required moment for recording has to be defined. Two cases are assumed:

1) The time demand of manufacturing process is known.

Therefore the simulation length is set according to the time

limit. Excel recording occurs after the limit expires.

2) Customer order size is known and therefore the simulation occurs after expiration of time inverval needed to complete the required number of products.

These solutions will be described further. Time savings are shown by an example,

Standard setting requires intermediate knowledge of the simulation program, so the user can simulate on the basis of order size or time demand. There are elements integrated in the model, which cause the software to be less demanding on operational skills.

In following parts of the article, there is a manufacturing system utilized. Basic character of this system is ilustrated by Fig. 1

First two systems are used to prepare semi-products delivered for finalization. Finalization is conducted by third system:

1)Cowls are pressed from granula material. Finished cowls are send for finalization to manufacturer, who varnishes them.

2)Adhesives are cut and send to manufacturer. who ensures their application.

3)Cowls are varnished and adhessives agglutinated.

Data exchange needs to be established between these three separated models. Two of them manufacture semi-products and third is used to manufacture the final product.

We assume a finished standard model created in PLANT SIMULATION. Next part of the article is focused on parameters settings, used to increase data record efficiency and improve user comfort. Complete model is at authors' disposal, however its' full description is complex an not provided in this paper.

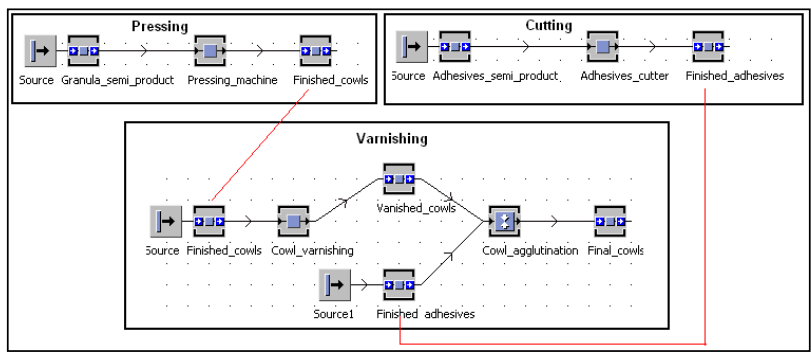

Fig. 1. Production system

\subsection{End-of-simulation recording}

If stationary simulation time interval is used (i.e. one shift), the recording can be conducted once, at the end of this interval. 
Through this adjustment a considerable amount of time is saved. Moreover the model allows novice person to operate it.

The Excel recording adjustment is conducted as follows:

Real type variable with assigned value of total planned simulation time is used. The variable is named sim_time and is further utilized to ensure required actions. Moreover, two methods are needed. First (counter) provides counting of finished products and second (writing) provides control of simulation time and activates Excel recording. TableFile named products is also set up. This TableFile is used to record the number of manufactured products into Excel.

Counter method contains the following text: is

do finished_quantity $:=$ finished_quantity +1 ;

@.move(finished_cowls);writing;end;

writing: is do if sim_time-60 < EventController.simTime then products $[1,2]:=$ finished_quantity;

Products.writeExcelFile("L:Yproducts.xlsx", "List1"); end;end;

These functionalities allow recording of number of manufactured products into Excel when simulation time reaches the value of sim_time variable lowered by machine cycle time (number 60 is important to capture the right moment for recording conduction).

Method for variable filling will be discussed further. The variable can be filled by input from Excel can be provided. This method is suitable for novice operator however it causes a considerable time delay. Time can be set up directly in the model by variable filling. However, this solution is complicated and time demanding. The simplification of the filling process is provided by PLANT SIMULATION feature called Dialog. When operator pushes simulation button, a dialog window pops up with request to set simulation period length.

Two methods are required for dialog control. First method opens the dialog window at the start of simulation and second method provides transformation of used input into adequate variable.

Method production is set in callback method by dialog.

It contains the following text: (action : string)is do inspect action when "Open" then eventcontroller.stop when "Close" then

simulation_time: $=$ str_to_num(dialog.getValue("demand")); sim_time:=simulation_time*60; eventcontroller.start

end;end;

This method ensures simulation termination until operator inserts his request. Then the simulation is reinitiated.

Writing method will be adjusted the following way (a text is inserted into eventcontroller.stop):is

do if sim_time-60< EventController.simTime

then products $[1,2]:=$ finished_quantity;

Products.writeExcelFile("L:|products.xlsx", "List1");

eventcontroller.stop; end;end;

This provides simulation termination at the end of simulation period. There is no need to limit this feature in eventController. Moreover the model operating speed is increased and requirements for operator qualification are reduced.

Time demand reduction is shown in chapter 2.3. Comparison is provided between version of consecutive recording and end-of-simulation period recording. Time reduction of end-of-simulation recording and consecutive recording is approximately $99 \%$.

\subsection{Recording on the basis of order size}

Simulation can often be conducted through customer order size. In this case model adjustments can be made as well. Furthermore there is a possibility to set up the model, in order to provide recording of number of manufactured products into Excel after all products are manufactured (not consecutively). Unlike the previous model version, only few changes are necessary. Variable is needed, to ensure the Dialog window is filled by number of required products.

Integer type variable demand is set. The filling is done by Dialog. The filling allows changes in model only after "Close" action is done.

After start of the simulation the operator inserts number of products that are to be manufactured. This number is assigned to variable representing number of products.

Therefore the writing method is adjusted to record the variable demand when it reaches finished_quantity value:

The writing method in cooperation with counter method allows Excel recording and simulation termination when the number of manufatured addhesives reaches the planned number.

Recording time demand in this case will be similar as in the case of end-of-simulation period recording.

\subsection{Contributions of the paper}

1)Standard model: recording of one variable into Excel at the end of every cycle, cycle time 1 minute, simulation time 100 minutes. Average time of simulation: 288 seconds

2)Advanced model: recording of one variable into Excel at the end of simulation time, cycle time 1 minute, simulation time 100 minutes. Average time of simulation: 3, 5 second.

Time reduction of advanced model is $98,78 \%$

\section{CONCLUSION}

Computer simulations provide optimization of manufacturing process. The need for optimization emerges with growing number of manufacturing systems which involve chain of subcontractors contributing to product completion. Thus a fast and simplified data exchange between individual manufacturing models is required. This article presents an option for meeting these requirements. Emphasis is put on simplicity of manipulation of the model. Therefore the paper holds a considerable potencial for expansion of final models among large number of users - for example, company planners can use these models to enhance efficiency of their manufacturing process. Through an example, the paper demonstrates time reduction of computer simulations by nearly $99 \%$. This reduction is dependant on specific model setting, simulation time and number of manufactured products. In extreme cases the contribution can be null, for example if only one product is manufactured. However these situations occur rarely. On the other hand, contributions are more significant if thousants of products with short processing time are manufactured. Time reduction provided by computer simulations in these cases reach almost $100 \%$ in comparison with standard setting.

\section{ACKNOWLEDGEMENTS}

Author is thankful to the Czech Science Foundation (GA CR) No. 402/08/H051 for financial support to carry out this investigation.

\section{REFERENCES}

Kumar, S, Nottestad, D, Macklin, J. (2007) A profit and loss analysis for male-to-order versus make-to-stock policy asupply chain case. Engineering Economist. vol. 52, no. 2 s. 141-156. ISSN0013-791X

Lassalle, S; Wang, Q; Owen, GW; Mileham, AR. (2007) A Study of In-process Waiting Time on a Linear Walking Worker Assembly Line. Proceedings of the Institution of Mechanical Engineers Part B-journal of Engineering Manufacture. vol. 221 , no. 12 s. 1763-1770. ISSN: 09544054 